\title{
Magnetic mechanism of quasiparticle pairing in hole-doped cuprate superconductors
}

\author{
R.S. Markiewicz and A. Bansil \\ Physics Department, Northeastern University, Boston MA 02115
}

(Dated: October 31, 2018)

\begin{abstract}
We have computed $\alpha^{2} F$ 's for the hole-doped cuprates within the framework of the one-band Hubbard model, where the full magnetic response of the system is treated properly. The d-wave pairing weight $\alpha^{2} F_{d}$ is found to contain not only a low energy peak due to excitations near $(\pi, \pi)$ expected from neutron scattering data, but to also display substantial spectral weight at higher energies due to contributions from other parts of the Brillouin zone as well as pairbreaking ferromagnetic excitations at low energies. The resulting solutions of the Eliashberg equations yield transition temperatures and gaps comparable to the experimentally observed values, suggesting that magnetic excitations of both high and low energies play an important role in providing the pairing glue in the cuprates.
\end{abstract}

PACS numbers: PACS number(s): 71.10.Fd 71.30.+h

\section{INTRODUCTION}

Since the superconducting state in the cuprates evolves from the doping of a Mott insulator, it is natural to conjecture that the pairing is driven by magnetic fluctuations rather than by phonons. Quantum Monte Carlo (QMC) calculations provide evidence for d-wave pairing [1, 2], where the pairing bosons reside predominantly in the (transverse) spin channel. Recent debate in this connection has centered on whether or not the magnetic resonance peak is strong enough to account for the condensation energy 3, 4]. Although recent estimates seem to be affirmative [5], they do not take into account competing pairbreaking effects which enter the Eliashberg equations [6]. Also, there are arguments that high-energy excitations play a role[7, 8, 9]. Here we report a computation of $\alpha^{2} F$ 's for the hole-doped cuprates based on the one-band Hubbard model, where the full magnetic response of the system is included, and the Eliashberg equations are then solved selfconsistently to obtain the superconducting properties over a wide range of dopings and temperatures. The resulting transition temperatures and pairing gaps are found to be comparable to experimental values, showing clearly the viability of the magnetic mechanism in the cuprates. We find that excitations at both high and low energies are important.

Early calculations of magnetic pairing in the cuprates employed parametrized models of the susceptibility. The analysis of Radtke, et al. (RULN) [10] invokes neutron scattering measurements, while that of Millis, et al. (MMP) 11] is based on NMR data. The model $\alpha^{2} F$ 's so obtained lead to divergent predictions concerning the feasibility of magnetic mechanism 12]. Our d-wave pairing weight $\alpha^{2} F_{d}$ contains not only a low-energy peak (LEP) from near- $(\pi, \pi)$ scattering, but also an additional high energy feature (HEF) extending to $\sim 1.5 \mathrm{eV}$ dominated by other regions of the Brillouin zone (BZ), as well as a significant pairbreaking contribution at low energies from ferromagnetic fluctuations. The HEF, which was missing in the RULN and MMP models turns out to be crucially important in producing high transition temperatures and pairing gaps. The pairbreaking terms begin to dominate as the Fermi energy approaches the van Hove singularity (VHS) with increasing doping and can lead to the loss of superconductivity.

Our study bears on the recently discovered 'waterfall' or high-energy kink (HEK) features observed over 0.3-1.2 $\mathrm{eV}$ range in the angle-resolved photoemission (ARPES) spectra of a number of cuprates. The magnetic susceptibility underlying our computation of $\alpha^{2} F$ 's yields self-energies and dispersions consistent with the waterfall effects [13, 14], suggesting that the boson responsible for the waterfall effects is also a key player in generating significant pairing weight in $\alpha^{2} F_{d}$ and high condensation energy in the cuprates.

The calculations are based on a one-band Hubbard Hamiltonian, extended to include pairing interaction. Specifically, in terms of susceptibility $\chi_{0}$ and the Hubbard on-site repulsion $U$, we use the singlet pairing potential [15]

$$
V_{s}=\frac{U}{1-U^{2} \chi_{0}^{2}\left(p^{\prime}-p\right)}+\frac{U^{2} \chi_{0}\left(p^{\prime}+p\right)}{1-U \chi_{0}\left(p^{\prime}+p\right)}
$$

and the mass renormalization potential (Eq. [8] of Ref. 15(a))

$$
V_{z}=\frac{U^{2} \chi_{0}\left(p^{\prime}-p\right)}{1-U^{2} \chi_{0}^{2}\left(p^{\prime}-p\right)}+\frac{U^{3} \chi_{0}^{2}\left(p^{\prime}-p\right)}{1-U \chi_{0}\left(p^{\prime}-p\right)},
$$

where $p$ and $p^{\prime}$ are the electron momenta, which are constrained to lie on the Fermi surface. Here $V_{z}\left[V_{s}\right]$ is the potential contributing to the normal [anomalous] part of the self energy. These expressions have been found to give transition temperatures in good agreement with QMC results [2]. The resulting coupling constants in various pairing channels $\alpha$ are

$$
\bar{\lambda}_{\alpha}=-\iint d^{2} p d^{2} p^{\prime} \tilde{g}_{\alpha}(p) \tilde{g}_{\alpha}\left(p^{\prime}\right) V\left(p, p^{\prime}, \omega=0\right)
$$

where $V=V_{s}$ for the even parity channels. The normalized weighting function $\tilde{g}_{\alpha}=g_{\alpha}(p) /\left(N_{0}\left|v_{p}\right|\right)$, where $v_{p}$ 


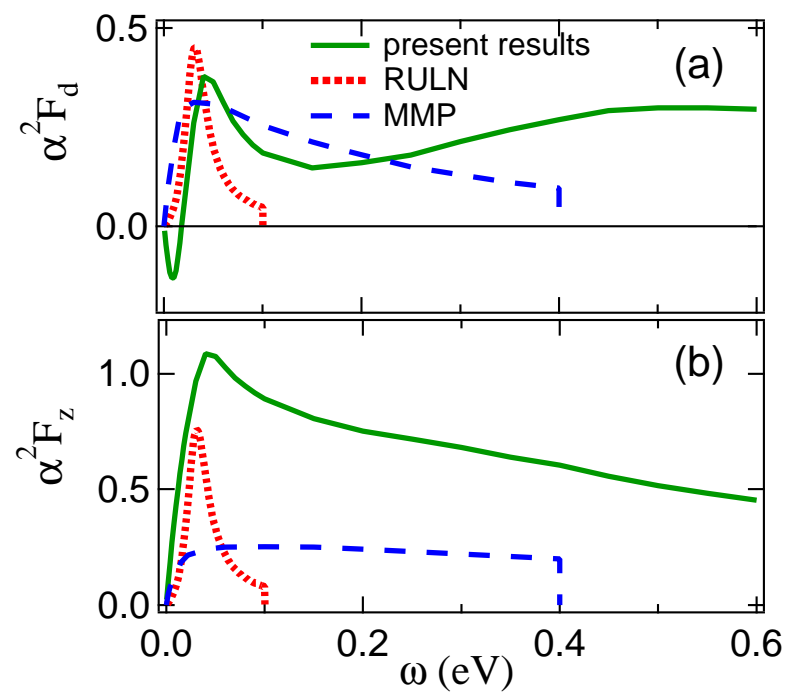

FIG. 1: (Color online) Eliashberg functions $\alpha^{2} F_{d}$ and $\alpha^{2} F_{z}$ for hole doping $x=0.30$ obtained in this work (green line) are compared with results of Refs. 10 (red dotted line) and 11 (blue dashed line).

is the Fermi velocity and $N_{0}^{2}=(2 \pi)^{3} \int g_{\alpha}(p)^{2} d^{2} p /\left|v_{p}\right|$. The $g_{\alpha}$ are weighting functions of various symmetry [15], of which the most important are the lowest harmonics of $s$-wave and $d_{x^{2}-y^{2}}$ symmetry, with $g_{s}=1$ and $g_{d}=\cos \left(p_{x} a\right)-\cos \left(p_{y} a\right)$. We also define the coupling constant $\lambda_{z}$ via the $s$-wave version of Eq. 3 with $V=V_{z}$. Then the effective BCS coupling is $\lambda_{\alpha}=\bar{\lambda}_{\alpha} /\left(1+\lambda_{z}\right)$. The symmetrized Eliashberg functions then are

$$
\alpha^{2} F_{\alpha}(\omega)=-\frac{1}{\pi} \iint d^{2} p d^{2} p^{\prime} \tilde{g}_{\alpha}(p) \tilde{g}_{\alpha}\left(p^{\prime}\right) V^{\prime \prime}\left(p, p^{\prime}, \omega\right),
$$

where $V^{\prime \prime}$ is the imaginary part of the corresponding $V$.

In the presence of strong magnetic fluctuations Migdal's theorem is not obeyed[16, 17]. We have developed a relatively simple approximation scheme [13, 18] which can successfully reproduce the pseudogap and waterfall phenomena in the normal state of the cuprates over the full doping range. In the overdoped regime, this scheme reduces to calculating the self-energy in GW approximation, using a reduced $U=3.2 t$ and dispersion renormalized via $Z_{0}=2$.21] These values of $U$ and $Z_{0}$ yield self-energies in reasonable accord with the QMC results 22 and explain the recently observed waterfall effects in photoemission spectra of the cuprates. [13, 14] We therefore expect these parameters to be most appropriate near $x=0.27$, but to gain some understanding of how the band structure would affect superconductivity in the absence of pseudogap effects, we solved the Eliashberg equations over the full doping range $x=0-0.4$, assuming $Z_{0}$ and $U$ to be doping independent. A more satisfactory procedure would be to let $U$ increase in the underdoped regime. However, in the presence of a pseudogap, a tensor system of Eliashberg equations needs to be solved, and that is beyond the scope of the present calculation. In short, we proceed thus by solving Eqs. 1 and 2 using $U=3.2 t$ and $\chi_{0}$ renormalized by $Z_{0}$. In particular, we neglect the additional modifications of Migdal's theorem in the superconducting state. Despite this limitation, our results provide a benchmark for the Eliashberg formulation in that we do not invoke empirical susceptibilities as has been the case in much of the existing literature.

Concerning technical details, we use a tight-binding parametrization of the dispersion of Bi2212, with the bilayer splitting neglected.[19] $\chi_{0}$ is first computed within the RPA scheme throughout the BZ for frequencies up to $2.88 \mathrm{eV} . \alpha^{2} F$ 's and the $\lambda$ 's are then computed from Eqs. 1-4. Fermi surface restricted Eliashberg equations [10] are finally used to selfconsistently obtain the gap $\Delta(\omega)$ and renormalization $Z(\omega)$ functions, with $Z(0) \equiv Z=$ $1+\lambda_{z} \cdot 20$

\section{PURE D-WAVE SOLUTION}

Figure 1, which compares our typical d-wave pairing weights $\alpha^{2} F_{d}$ and $\alpha^{2} F_{z}$ with RULN and MMP models, highlights our key finding. Our $\alpha^{2} F_{d}$ (green line) in (a) displays two clear features [23]: A low energy peak (LEP) around $40 \mathrm{meV}$ and a broad high energy hump-like feature (HEF) extending from $\sim 0.5-1.0 \mathrm{eV}$ (see also Fig. 2(a) below). The LEP arises mainly from the magnetic response near $(\pi, \pi)$, but the HEF is connected with the response from other parts of the BZ, particularly near $(\pi, 0)$ and $(\pi / 2, \pi / 2)$. Our LEP in (a) is similar to the weights assumed by RULN and MMP. This resemblance is not surprising since the RULN model[10] was designed to match neutron scattering data near $(\pi, \pi)$, while the NMR data utilized by MMP [11] is also most sensitive to weight in this part of the BZ. It has long been known that neutron scattering near $(\pi, \pi)$ accounts for only about $1 / 8$ th of the integrated spectral weight expected from a total scattering sum rule 24]. By basing their estimate solely on the neutron scattering data near $(\pi, \pi)$, RULN severely underestimated the total d-wave pairing weight. The MMP analysis, based on NMR data, appears to have captured more of the weight-although still missing the HEF and thus underestimating the total weight. Note also from Fig. 1(b) that both models strongly underestimate the renormalization weight $\alpha^{2} F_{z}$, which opposes the tendency for pairing.

The negative dip in Fig. 1(a) at energies below $20 \mathrm{meV}$ deserves comment. This dip reflects pair-breaking magnetic scattering (PBS) near $\Gamma$ and was overlooked in the phenomenological RULN and MMP models. For simplicity, we will refer to these fluctuations as being ferromagnetic (FM), although this is strictly so only at $\Gamma$. This PBS is related to earlier indications of FM instabilities near a VHS 25, 26]. A similar scenario of competing dwave pairing vs pairbreaking effects has been discussed 


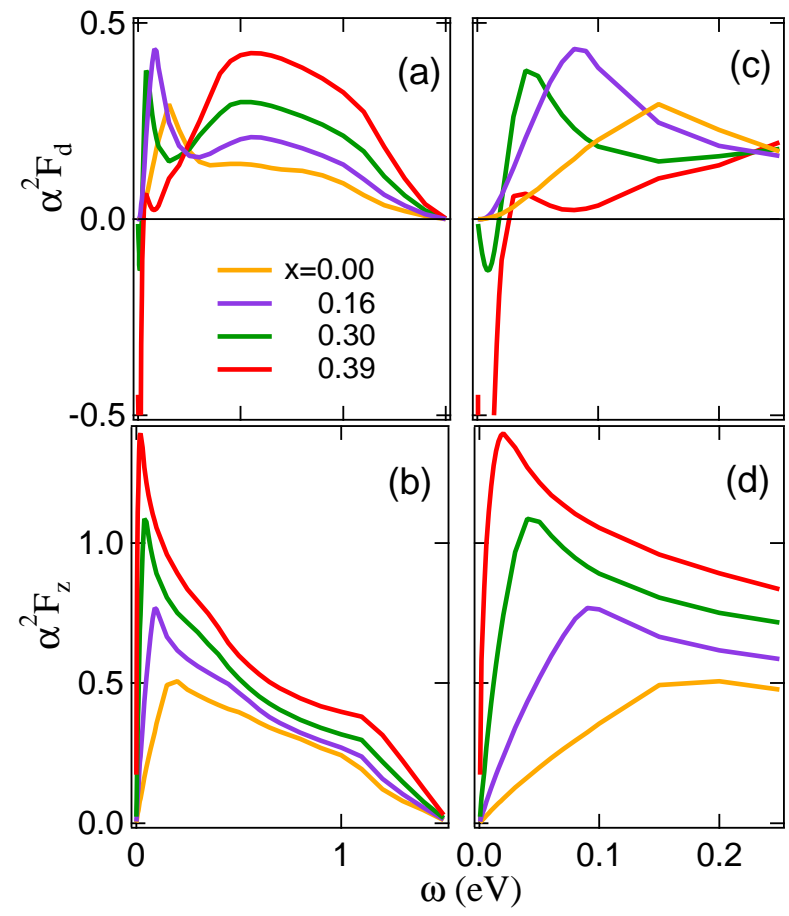

FIG. 2: (Color online) Eliashberg functions $\alpha^{2} F_{d}$ and $\alpha^{2} F_{z}$ over the doping range $x=0.0-0.4$. Lines of various colors refer to different dopings (see legend in (a)). Left hand panels (a) and (b) give results over an extended frequency range of $0-1.5 \mathrm{eV}$, while right hand panels (c) and (d) highlight the low energy region of $0-250 \mathrm{meV}$ on an expanded energy scale.

in the context of electron-phonon pairing 27 .

Figure 2 shows how $\alpha^{2} F$ 's evolve with doping. In (a), the pairing weight in the high energy feature of $\alpha^{2} F_{d}$ is seen to increase monotonically with increasing doping, displaying an approximate isosbestic point at $\omega \sim 0.24 \mathrm{eV}$. In the low energy region in (c), position of the peak in $\alpha^{2} F_{d}$ shifts to lower energies with increasing doping, and the negative pair breaking peak grows dramatically, consistent with the suggestion of Kopp et al.[26]. The nature of $\alpha^{2} F_{d}$ is seen to change quite substantially as the Fermi energy approaches the VHS at around $x=0.39$. Interestingly, by comparing (c) and (d), the low energy peak in $\alpha^{2} F_{z}$ is seen to follow that in $\alpha^{2} F_{d}$ to lower energies with doping.

Figure 3 shows the doping dependence of $\lambda_{z}, \lambda_{d}$ and the low temperature gap $\Delta_{d}(T=0)$. Three different estimates of $\lambda_{z}$ are compared in (a) for illustrative purposes. Values based on using the bare susceptibility, $V_{z 0}=U^{2} \chi_{0}$ (red dashed line), are seen to be quite similar to the simple estimate $N(0) U$ (green dotted line), where $N(0)$ is the density-of-states at the Fermi energy. The full $V_{z}$ (blue line) on the other hand yields a significant enhancement of $\lambda_{z}$ over that obtained from $\chi_{0}$, especially near the region of the VHS peak, indicating that the system is close to a magnetic instability. Note that $\lambda_{d}$ is positive for dopings less than $\approx 0.4$, but as the Fermi energy enters

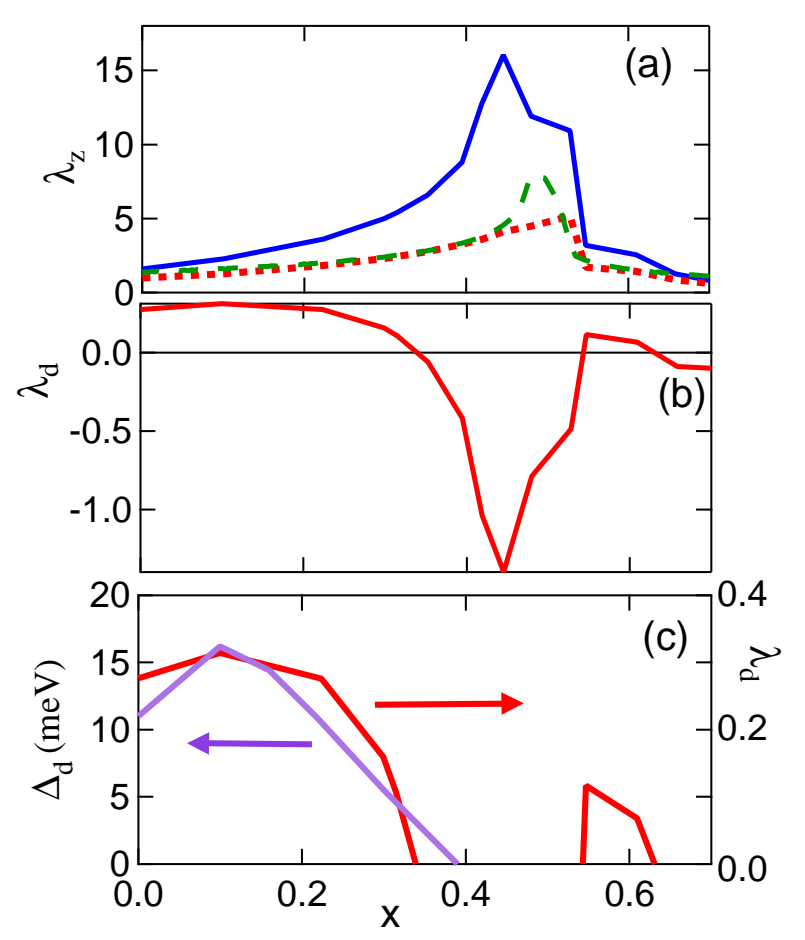

FIG. 3: (Color online) Doping dependence of: (a) $\lambda_{z}$; (b) $\lambda_{d}$; and (c) $\Delta_{d}(T=0)$ (left scale) compared to $\lambda_{d}$ (right scale). In (a) three different computations of $\lambda_{z}$ are compared based on the full $V_{z}$ of Eq. 2 (blue solid line), a simplified $V_{z 0}=U^{2} \chi_{0}$ (red dotted line), and the estimate $N(0) U$ (green dashed line), where $N(0)$ is the density-of-states at the Fermi energy.

the region of the VHS with increasing doping, $\lambda_{d}$ rapidly becomes large and negative due to FM fluctuations. (c) shows that this doping dependence of $\lambda_{d}$ is well correlated with that of the pairing gap. We stress that these results hold for a pure $d_{x^{2}-y^{2}}$ order parameter. Harmonic content plays an important role, as will be discussed below, Section III.

We turn now to discuss our solutions of the Eliashberg equations. Following common practice, we proceeded by discretizing the $\alpha^{2} F$ 's on the real frequency axis. [28] We find that our results are sensitive to the number $N_{m}$ of points in the mesh. For the present calculations, based on a 768 -point non-uniform mesh over $0-2.88 \mathrm{eV}$, the gap $\Delta(\omega)$ is approximately converged in the low- $\omega$ regime, allowing us to extract $\Delta_{d}(T)$. Figure 4 (a) shows typical results for the real part of $\Delta(\omega)$ for a range of temperatures at $x=0.10$. The prominent oscillations in $\Delta(\omega)$ curves are the well-known consequence of discretizing $\alpha^{2} F$ 's in solving the Eliashberg equations. 29] We define the gap by taking the intersection of the $\Delta(\omega)=\omega$ line (thin black line in Fig. 4(a)) with the $\Delta(\omega)$ curve.

Fig. 4(b) shows how the computed low-energy gap $\Delta_{d}$ evolves with temperature at various dopings. Due to the difficulty of finding well-converged solutions when $\Delta$ is small, we calculate $\Delta_{d}(T)$ at a few low temperatures, and use a fit to a $d$-wave BCS gap to estimate $T_{c}$. We find 

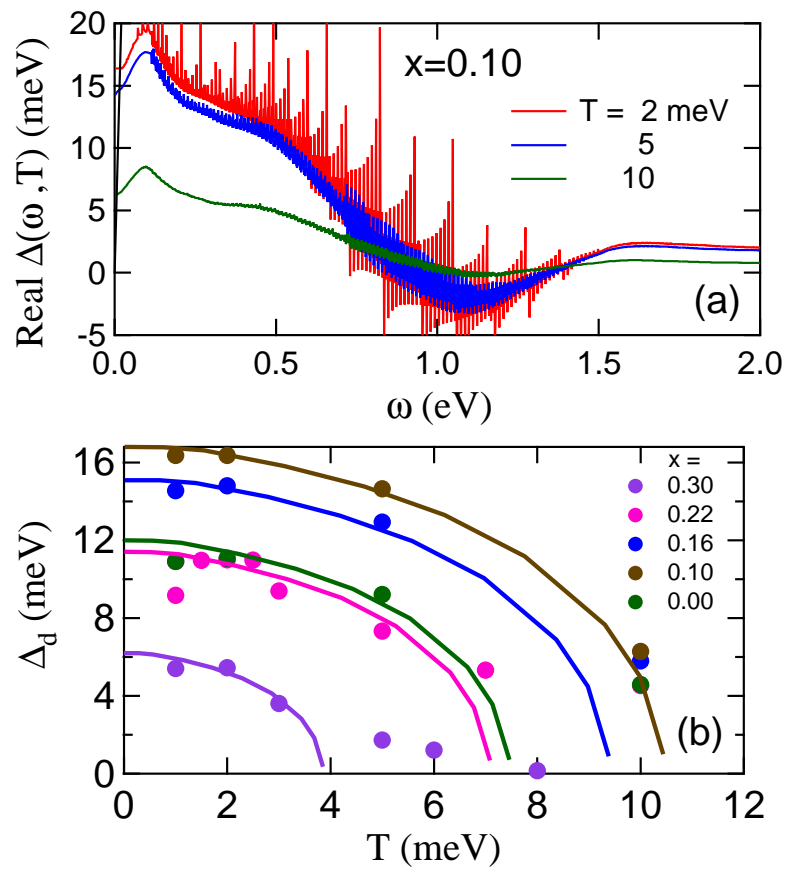

FIG. 4: (Color online) (a) Real part of the gap function $\Delta(\omega, T)$ at doping $x=0.10$ as a function of frequency for a series of temperatures $\mathrm{T}$ (see legend). Thin black line is the plot of $\Delta=\omega$ used to obtain the low energy gap as discussed in the text. (b) Computed temperature dependence of the low-energy gap $\Delta_{d}(T)$ at various dopings $x$ (see legend).

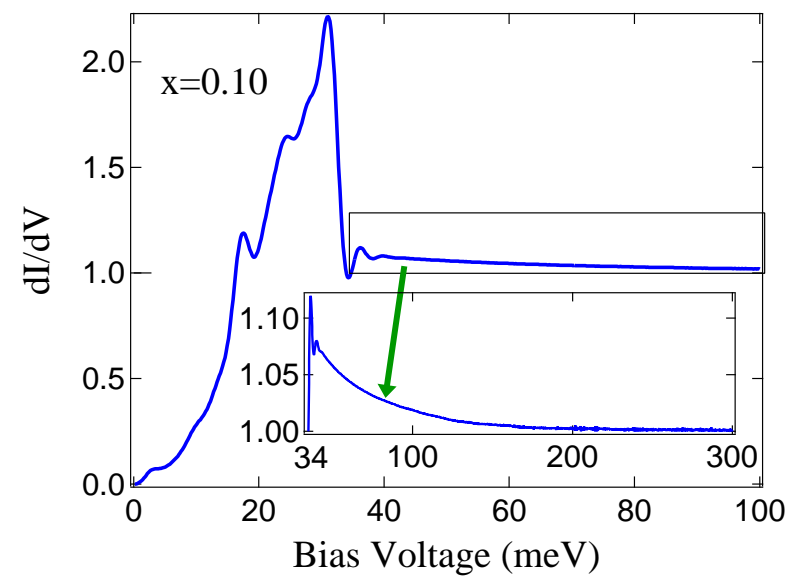

FIG. 5: (Color online) Typical computed SIS tunneling spectrum at $x=0.10$. Inset shows the high energy tail on an expanded scale.

$2 \Delta_{d}(0) / k_{B} T_{c} \sim 3.2$ for different dopings. The resulting $\mathrm{T}_{c}$ 's are somewhat smaller than QMC values [2], perhaps the effect of a finite $t^{\prime}$.

It is striking that the gap features in Fig. 4(a) extend to very high energies, raising the obvious question as to how this high-energy tail would show up in tunneling spectra. 30. Insight in this regard is provided by Fig. 5, where we show a typical tunneling spectrum computed 31] within our model. [Tunneling spectra computed at other dopings are similar, except that the features scale with $\Delta_{d}$.] The weight in Fig. 5 at energies above the peak-dip-hump feature is seen to be quite small with weak energy dependence (see inset) and would not be readily observable in the presence of an experimental background.

\section{LOW VS HIGH ENERGY PAIRING GLUE}

Within the present model, the LEP and HEF both play an essential role in generating large gaps. For example, at $x=0.3$, the HEF by itself produces a gap of only $\sim$ $0.4 \mathrm{meV}$, while the LEP is virtually nonsuperconducting, even though the full $\alpha^{2} F_{d}$ yields a gap of $5.5 \mathrm{meV}$. [To be definite, we separate $\alpha^{2} F$ into LEP and HEF at the minimum in $\alpha^{2} F, \omega_{\text {min }}=0.3 \mathrm{eV}$.] Similarly, for $x=0.1$, LEP [HEF] by itself has a $\sim 3[0.4] \mathrm{meV}$ gap, with a combined gap of $\sim 17 \mathrm{meV}$, with $\omega_{\min }=0.16 \mathrm{eV}$.

This behavior can be readily understood from a $2-\lambda$ model. 32 Since this is a purely electronic mechanism, we use a modified Allen-Dynes formula 33, 34]

$$
T_{c}=\frac{\omega_{l n}}{1.2} \exp \left(\frac{-1.04\left(1+\lambda_{z}\right)}{\bar{\lambda}_{d}}\right)=\frac{\omega_{l n}}{1.2} \exp \left(\frac{-1.04}{\lambda_{d}}\right),
$$

$\Delta(0)=3.54 T_{c}$, with

$$
\bar{\lambda}_{d}=2 \int_{0}^{\infty} \frac{\alpha^{2} F(\omega)}{\omega}
$$

and

$$
\ln \left(\omega_{l n}\right)=\frac{2}{\bar{\lambda}_{d}} \int_{0}^{\infty} \ln (\omega) \frac{\alpha^{2} F(\omega)}{\omega}
$$

The Allen-Dynes equation has a well-known limitation [34] that it predicts a maximum $T_{c}=\omega_{l n} / 1.2$, whereas the Eliashberg equations have a solution that grows without limit $\sim \sqrt{\bar{\lambda}}$ as $\bar{\lambda} \rightarrow \infty$. We find that this leads to an underestimate of $\Delta_{L E P}$, while the model provides good estimates for the remaining gaps. For instance, for $x=0.1, \lambda_{L E P}=\lambda_{H E F}=0.15$, $\omega_{l n, L E P}=83 \mathrm{meV}, \omega_{l n, H E F}=530 \mathrm{meV}$, so $\Delta_{L E F}=0.26 \mathrm{meV}$, and $\Delta_{H E F}=1.4 \mathrm{meV}$. When both features are combined, $\omega_{l n}=200 \mathrm{meV}$ and $\lambda_{d}=0.3$, leading to $\Delta_{d}=19 \mathrm{meV}$, in good agreement with the full calculation. While the Allen-Dynes model is highly simplified, it does capture the observed trend that both peaks contribute significantly. Physically, the effective $\lambda$ is in the weak coupling regime, $\lambda \sim<<1$, so high $T_{c}$ arises from the large $\omega_{l n}$, and the large boost from combining LEP and HEF arises since $e^{-1 / 2 \lambda}>>2 e^{-1 / \lambda}$. Clearly, an electron-phonon coupling could play a similar role in further enhancing $T_{c}$. 


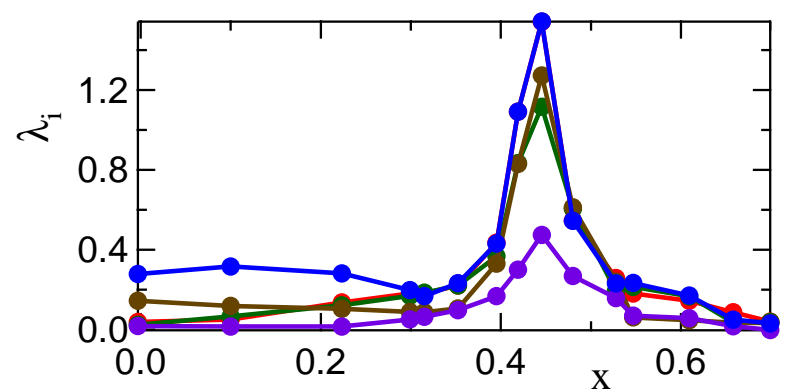

FIG. 6: (Color online) Doping dependence of: $\lambda_{d}$ (blue line), $\lambda_{s}$ (red line), $\lambda_{d x y}$ (green line), $\lambda_{p}$ (violet line), and $\lambda_{s p}$ (brown line), calculated from a $15 \times 15$ harmonic matrix in each symmetry sector.

\section{COMPETING ORDER PARAMETER SYMMETRIES}

The above calculations have been limited to a pure $d$-wave gap symmetry, without harmonic content. In tetragonal symmetry there are five symmetry classes of superconducting gap, and each class can involve higher harmonics of the given symmetry [35]. While we have not solved the tensor Eliashberg equations, it is straightforward to generalize the $\lambda$ calculations to include harmonic structure and to calculate the leading $\lambda$ eigenvalue for each symmetry class. The results are shown in Fig. [ following the analysis of Ref. 36. We see that: (1) The pure$d$ analysis of Section II holds in the low doping regime; (2) Near the VHS, harmonic content stabilizes d-wave symmetry, leading to the largest gaps; (3) In this regime, other symmetries can become comparable to d-wave. In particular, there is a tendency toward $s$-wave pairing in the overdoped case.

\section{CONCLUSIONS}

In summary, we have shown that within the present model the d-wave pairing weights $\alpha^{2} F_{d}$ and $\alpha^{2} F_{z}$ extend to very high energies of $\sim 1 \mathrm{eV}$ when the magnetic response of the system is properly taken into account. The associated superconducting gap is quite substantial, being around $16 \mathrm{meV}$ at low dopings. $\alpha^{2} F_{d}$ is found to contain not only the expected low energy peak (LEP) below $200 \mathrm{meV}$, but a previously unrecognized high energy feature (HEF) over 0.3-1.2 eV. We find that the LEP and HEF both play an important role in yielding a large gap in our model. This suggests that electron-phonon coupling could be important for further enhancing $T_{c}$ as suggested by the isotope effect 37]. The gap spectrum $\Delta(\omega)$ generally extends to the limit of $\alpha^{2} F(\sim 1.5 \mathrm{eV})$, and may provide insight into a number of anomalous features connected with optical properties of the cuprates, summarized for example in Ref. 38 .
The scarcity of high- $T_{c}$ superconductors arises in part from the fact that when superconducting pairing is sufficiently strong, corresponding and stronger instabilities arise in other channels. We have for the most part neglected the effects of competing phases, but it is clear that they will be significant, both in the underdoped regime and near the VHS. Near the VHS pairbreaking ferromagnetic scattering increases sharply, strongly suppressing a pure $d$-wave gap. While we find that inclusion of harmonic content could stabilize a d-wave superconductor even at the VHS, we have not accounted for a competing FM instability. Indeed, Storey, et al. [39] find that in Bi2212 the VHS induces strong pair breaking, suppressing superconductivity, so that optimum $T_{c}$ falls at a doping below the VHS. This is consistent with the evidence for strong FM pairbreaking adduced by Kopp et al. [26]

To conclude, we have demonstrated that, when realistic $\alpha^{2} F$ 's are used to solve the Eliashberg equations, the magnetic mechanism is capable of producing transition temperatures and pairing gaps comparable in size to those observed experimentally in the cuprates. The low values of these key superconducting properties found in earlier calculations are directly attributable to the fact that neutron scattering sees only a fraction of the total magnetic spectral weight in these materials.

It is a pleasure to acknowledge useful discussions with Mark Jarrell. This work is supported by the US Department of Energy, Basic Energy Sciences, Division of Material Science and Engineering contract DE-FG0207ER46352 and benefited from the allocation of supercomputer time at NERSC and Northeastern University's Advanced Scientific Computation Center (ASCC).

[1] T.A. Maier, M. Jarrell, and D.J. Scalapino, Phys. Rev. B74, 094513 (2006).

[2] T.A. Maier, A. Macridin, M. Jarrell, and D.J. Scalapino, Phys. Rev. B76, 144516 (2007).

[3] E. Demler and S.-C. Zhang, Nature 396, 733 (1998); P. Dai et al., Science 284, 1344 (1999).

[4] H.-Y. Kee, S.A. Kivelson, and G. Aeppli, Phys. Rev. Lett. 88, 257002 (2002).

[5] H. Woo, P. Dai, S.M. Hayden, H.A. Mook, T. Dahm, D.J. Scalapino, T.G. Perring, and F. Dogan, Nature Physics 2, 600 (2006).

[6] G.M. Eliashberg, Sov. Phys. JETP 11, 696 (1960).

[7] P.W. Anderson, Science 316, 1705 (2007).

[8] T.A. Maier, D. Poilblanc, and D.J. Scalapino, Phys. Rev. Lett. 100, 237001 (2008).

[9] S. Chakraborty, D. Galanakis, and P. Phillips, arXiv:0807.2854

[10] R.J. Radtke, S. Ullah, K. Levin, and M.R. Norman, Phys. Rev. B46, 11975 (1992).

[11] A.J. Millis, H. Monien, and D. Pines, Phys. Rev. B42, 167 (1990).

[12] H.-B. Schüttler and M.R. Norman, Phys. Rev. B54, 13295 (1996). 
[13] R.S. Markiewicz, S. Sahrakorpi, and A. Bansil, Phys. Rev. B76, 174514 (2007),

[14] A. Macridin, M. Jarrell, T. Maier, and D.J. Scalapino, Phys. Rev. Lett. 99, 237001 (2007).

[15] (a) D.J. Scalapino, E. Loh, Jr., and J. E. Hirsch, Phys. Rev. B34, 8190 (1986); (b) 35, 6694 (1987).

[16] P. Monthoux, Phys. Rev. B68, 064408 (2003).

[17] S. Moukouri, S. Allen, F. Lemay, B. Kyung, D. Poulin, Y.M. Vilk, and A.-M.S. Tremblay, Phys. Rev. B61, 7887 (2000).

[18] T. Das, R.S. Markiewicz, and A. Bansil, arXiv:0807.4257.

[19] The hopping parameters are taken from Ref. 13: $\left(t, t^{\prime}, t^{\prime \prime}, t^{\prime \prime \prime}\right)=(360,-100,35,10) \mathrm{meV}$.

[20] This BCS renormalization factor is the inverse of that normally used in Green function theory.

[21] We use $Z_{0}$ to distinguish the renormalization factor used in calculating $\chi_{0}$; it is not identical to the doping dependent $Z$ found from the Eliashberg equations.

[22] T.A. Maier, M. Jarrell, and D.J. Scalapino, Phys. Rev. B75, 134519 (2007).

[23] Calculated $\alpha^{2} F$ 's are mainly odd in $\omega$; a small even component was subtracted out in the displayed results.

[24] H.-Y. Kee, S.A. Kivelson, and G. Aeppli, Phys. Rev. Lett. 88, 257002 (2002); J. Lorenzana, G. Seibold, and R. Coldea, Phys. Rev. B72, 224511 (2005); C. Stock, W.J.L. Buyers, Z. Yamani, Z. Tun, R.J. Birgeneau, R. Liang, D. Bonn, and W.N. Hardy, Phys. Rev. B77, 104513 (2008).

[25] R. Hlubina, S. Sorella, and F. Guinea, Phys. Rev. Lett. 78, 1343 (1997).

[26] A. Kopp, A. Ghosal, and S. Chakravarty, Proc. Natl. Acad. Sci. USA 104, 6123 (2007).
[27] N. Bulut and D. J. Scalapino, Phys. Rev. B54, 14971 (1996).

[28] A.E. Karakozov, E.G. Maksimov, and A.A. Mikhailovsky, Sol. St. Commun. 79, 329 (1991).

[29] F. Marsiglio, M. Schossmann, and J.P. Carbotte, Phys. Rev. B37, 4965 (1988).

[30] J.F. Zasadzinski, L. Ozyuzer, L. Coffey, K.E. Gray, D.G. Hinks, and C. Kendziora, Phys. Rev. Lett. 96, 017004 (2006).

[31] L. Coffey, cond-mat/0103518.

[32] F. Marsiglio and J.P. Carbotte, Phys. Rev. B36, 3937 (1987).

[33] P.B. Allen and R.C. Dynes, Phys. Rev. B12, 905, (1975).

[34] J.P. Carbotte, Rev. Mod. Phys. 62, 1027 (1990).

[35] R. Hlubina, Phys. Rev. B59, 9600 (1999).

[36] F. Guinea, R.S. Markiewicz, and M.A.H. Vozmediano, Phys. Rev. B69, 054509 (2004).

[37] G.-H. Gweon, T. Sasagawa, S.Y. Zhou, J. Graf, H. Takagi, D.-H. Lee, and A. Lanzara, Nature, 430, 187 (2004); R. Khasanov, A. Shengelaya, D. Di Castro, D.G. Eshchenko, I.M. Savic, K. Conder, E. Pomjakushina, J. Karpinski, S. Kazakov, and H. Keller, Phys. Rev. B75, 060505(R) (2007).

[38] H.J.A. Molegraaf, C. Presura, D. van der Marel, P.H. Kes, and M. Li, Science 295, 2239 (2002); A.F. Santander-Syro, R.P.S.M. Lobo, N. Bontemps, Z. Konstantinovic, Z.Z. Li, and H. Raffy, Europhys. Lett. 62, 568 (2003).

[39] J.G. Storey, J.L. Tallon, and G.V.M. Williams, Phys. Rev. B76, 174522 (2007). 